\title{
A DENT IN THE FOREHEAD
}

\author{
Charles B. Davenport
}

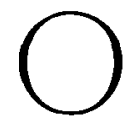

NE of the most striking phenomena to a student of heredity is the definiteness with which certain small, apparently insignificant, peculiarities are inherited. This is very prettily illustrated again by a case which a correspondent has sent to me and, although the family history is only a fragment, it is so instructive as to be worth publishing.

The trait in question consists of a small depression in the sagittal plane of the frontal bone, extending two or three centimeters above a line joining the upper limits of the orbits. The depression is a striking one, but the morphological changes involved do not seem to be great. Nevertheless they are very persistent in the family history and without doubt indicate a definite modification of the germ plasm which is, in accordance with the modern interpretation, of the positive, dominant sort; that is, due to the addition of a gene.

We start with a fraternity of three grown children, born between 1885 and 1890 (III, 20-23). The first, a male, has a deep dent in his forehead, as if his skull were pushed in. This is the young man whose photograph is presented herewith (Fig. 6). The second, male, has a deep crease or dent in forehead and the third, female, has a crease in forchead. A fourth member of the fraternity was a small boy who died at the age of nine months and of whom $\dot{w} e$ have no description. The father of this fraternity of three (II, 15) had a dent in the forehead. The mother (II, 14) was unrelated to the father and none of her relatives had a peculiarity of this sort. By another wife the father had one daughter (III, 24) who, likewise, had the crease in the forehead. As this other wife was unrelated and did not show the crease in the forehead, this portion of the pedigree, alone, is sufficient to suggest strongly that the positive trait is carried in the germ plasm of the father.

The father was one of a fraternity of seven, three males and four females. One of his brothers (II, 5) showed the same crease in the forehead. The other did not. Three of the sisters certainly have shown the crease in the forehead. One of these is married and had six children, one boy and five girls, and of these girls three show the crease in the forehead (III, 3-8). Of the fourth sister of the father, long since dead (III, 7), there are no precise data. In response to an inquiry my correspondent has examined a group photograph in which the sister appears and replies: "It is impossible to be sure, but I think there was a slight trace of it from what I could see." This fourth sister has had four children (by three husbands), and one (a son) shows the crease in the forehead. The single member of the second generation who lacks the family trait has two children, neither of whom has the trait; however, the same is true of the two children of his sister (II, 1) who has the trait. Unfortunately definite knowledge is extant concerning two generations only. The conditions in the grandparents are unknown. So far, then, as our information goes it indicates that this slight family peculiarity in the form of the frontal bone of the skull is inherited as a dominant trait.

In conclusion the writer wishes first to thank Miss Rose M. Dawson for her coopperation and, second, to request those in whose family such (or other clearly marked) peculiarity in the form of the skull, appears, or who know of such a family trait among their acquaintances, to communicate the fact to him.

Carnegie Institution of Washington, Station for Exper:mental Evolution, Cold Spring Harbor, Long Island, N. Y., Jan. 30, 1915. 


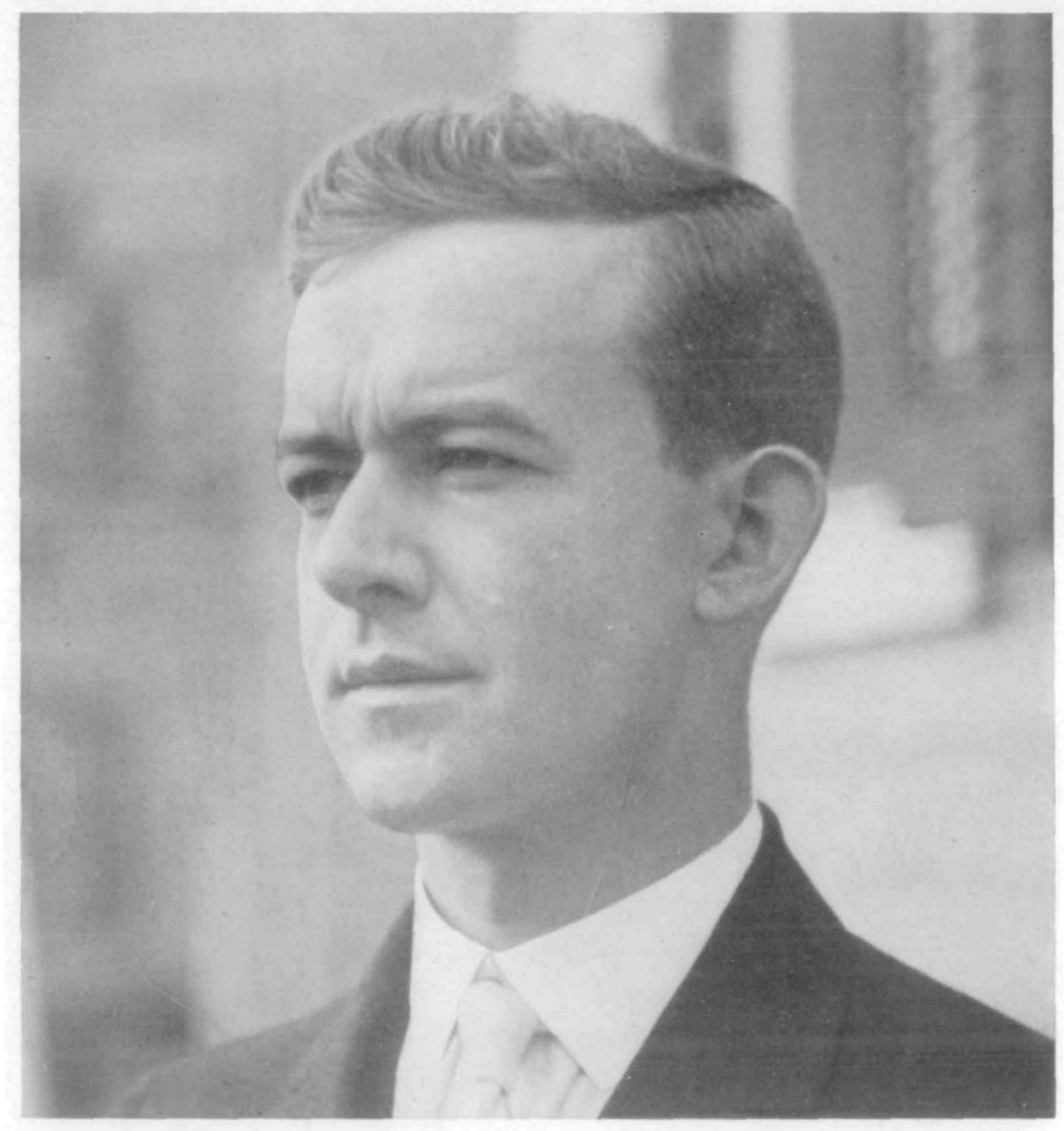

\section{A HERITABLE DENT IN THE FOREHEAD}

This young man is shown as III, 20, in the pedigree chart below. The dent in his skull is located in the median line of the forehead, at the upper end of the frown, and because of the illumination is much less conspicuous in the photograph than in real life. From a photograph copyrighted by Brown and Dawson. (Fig. 6.)

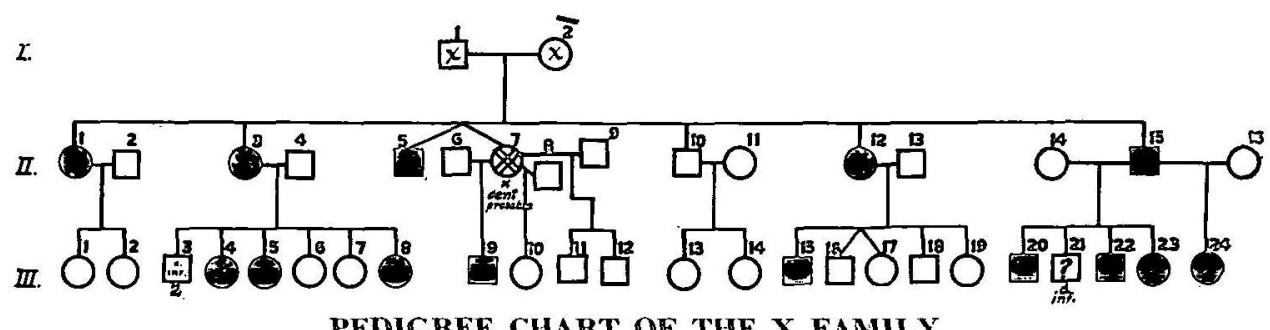

PEDIGREE CHART OF THE $X$ FAMLY

Distribution of the dent or depression in the forehead is here shown in graphic form. Squares represent males and circles females: black symbols designate individuals who show the trait in question; $\mathrm{x}$ implies unknown; the abbreviation d.inf. means "died in infancy." Some doubt exists as to whether individual No. 7 in generation II showed the trait or not. (Fig. 7.) 